\title{
Light scalar dark matter extension of the type-II two-Higgs-doublet model
}

\author{
Lei Wang, Rongle Shi, Xiao-Fang Han, ${ }^{*}$ and Bin Zhu \\ Department of Physics, Yantai University, Yantai 264005, China
}

(Received 15 March 2018; revised manuscript received 6 July 2018; published 16 August 2018)

\begin{abstract}
We examine the type-II two-Higgs-doublet model with a light scalar dark matter $(S)$ after imposing the constraints from the Higgs searches at the LHC and dark matter experiments. We first assume that both $C P$-even Higgses ( $h$ and $H$ ) are portals between the dark matter and standard model (SM) sectors, and the $C P$-odd Higgs $(A)$ and $H$ are heavier than $130 \mathrm{GeV}$. We find that the dark matter with a mass of $10-50 \mathrm{GeV}$ is disfavored by the joint constraints of the $125 \mathrm{GeV}$ Higgs signal data, the relic density, XENON1T (2018), and Fermi-LAT. Next, we consider a special scenario in which the heavy $C P$-even Higgs is taken as the $125 \mathrm{GeV}$ Higgs. The light $C P$-even Higgs is the only portal between the dark matter and SM sectors, and the dark matter mass is slightly below the Higgs resonance. We find that the signal data of the $125 \mathrm{GeV}$ Higgs restrict $\tan \beta$ to be in the range of 1-1.5 for $m_{h}<62 \mathrm{GeV}$. The $g g \rightarrow A \rightarrow h Z$ and $b \bar{b} \rightarrow h \rightarrow \tau^{+} \tau^{-}$ channels at the LHC can impose lower limits and upper limits on $\tan \beta$, respectively. For appropriate values of $\tan \beta, \lambda_{h}$, and $m_{h}$, the dark matter with a mass of $10-50 \mathrm{GeV}$ is allowed by the constraints of the Higgs searches at the LHC and dark matter experiments. For example, $\tan \beta$ is restricted to be in the range of $1.0-1.5$ for $10 \mathrm{GeV}<m_{s}<28 \mathrm{GeV}$, and $\frac{m_{h}}{2 m_{S}}>1.12$ is excluded for $30 \mathrm{GeV}<m_{S}<50 \mathrm{GeV}$.
\end{abstract}

DOI: 10.1103/PhysRevD.98.035024

\section{INTRODUCTION}

A weakly interacting massive particle (WIMP) is one of the most popular dark matter (DM) candidates. The simplest WIMP-DM model is the standard model (SM) plus a real singlet scalar as DM [1]. In the model, the current experiments exclude a DM mass up to $330 \mathrm{GeV}$, except for a small range near $63 \mathrm{GeV}[2,3]$. Much of the region in this model can be recovered if the Higgs sector is extended to the two-Higgs-doublet model (2HDM) [4] which contains two neutral $C P$-even Higgs bosons $h$ and $H$, one neutral pseudoscalar $A$, and two charged Higgs $H^{ \pm}$.

The type-II 2HDM with a singlet scalar as DM has been widely explored [3,5-13]. Recently, the authors of Ref. [3] showed that in such a model, much of the DM mass region below $100 \mathrm{GeV}$ is excluded if the heavy $C P$-even Higgs boson is the only portal between the DM and SM sectors. The authors of Ref. [12] took the $125 \mathrm{GeV}$ Higgs with a wrong-sign Yukawa coupling of the down-type quark as the only portal, and found that the DM mass is allowed to be as low as $50 \mathrm{GeV}$ for appropriate isospin-violating DM interactions with nucleons. Because of the tension between

\footnotetext{
* Corresponding author. xfhan@mail.itp.ac.cn

Published by the American Physical Society under the terms of the Creative Commons Attribution 4.0 International license. Further distribution of this work must maintain attribution to the author(s) and the published article's title, journal citation, and DOI. Funded by SCOAP .
}

the DM relic density and the invisible decay of the $125 \mathrm{GeV}$ Higgs, a DM mass below $50 \mathrm{GeV}$ is excluded. As the DM mass increases from 50 to $62.5 \mathrm{GeV}$, the width of the invisible decay becomes small due to phase-space suppression. Meanwhile DM pair annihilation approaches the resonant condition $\left(2 m_{\mathrm{DM}} \sim 125 \mathrm{GeV}\right)$. As a result, the tension is automatically relaxed. In this paper, we manage to find some parameter spaces where DM with a mass below $50 \mathrm{GeV}$ is allowed by the relic density and the invisible decay of the $125 \mathrm{GeV}$ Higgs. Also we will consider joint constraints from theory, precision electroweak data, flavor observables, the signal data of the $125 \mathrm{GeV}$ Higgs, the searches for the additional Higgses at LEP and the LHC, XENON1T (2018), and the FermiLAT searches for DM annihilation from dwarf spheroidal satellite galaxies (dSphs).

This paper is organized as follows. In Sec. II, we introduce some characteristic features of the type-II 2HDM with scalar DM. In Sec. III, we perform numerical calculations. In Sec. IV, we examine the allowed parameter space after imposing the relevant theoretical and experimental constraints. Finally, we draw our conclusions in Sec. V.

\section{TYPE-II TWO-HIGGS-DOUBLET MODEL WITH SCALAR DARK MATTER}

\section{A. Type-II two-Higgs-doublet model}

In the type-II 2HDM with scalar DM, the scalar potential includes two parts: $\mathcal{V}_{2 H D M}$ and $\mathcal{V}_{S}$. They are respectively the potential of the type-II $2 \mathrm{HDM}$ and the potential of the 
DM sector. $\mathcal{V}_{2 H D M}$ with a softly broken discrete $Z_{2}$ symmetry is given by [14]

$$
\begin{aligned}
\mathcal{V}_{2 H D M}= & m_{11}^{2}\left(\Phi_{1}^{\dagger} \Phi_{1}\right)+m_{22}^{2}\left(\Phi_{2}^{\dagger} \Phi_{2}\right)-\left[m_{12}^{2}\left(\Phi_{1}^{\dagger} \Phi_{2}+\text { H.c. }\right)\right] \\
& +\frac{\lambda_{1}}{2}\left(\Phi_{1}^{\dagger} \Phi_{1}\right)^{2}+\frac{\lambda_{2}}{2}\left(\Phi_{2}^{\dagger} \Phi_{2}\right)^{2}+\lambda_{3}\left(\Phi_{1}^{\dagger} \Phi_{1}\right)\left(\Phi_{2}^{\dagger} \Phi_{2}\right) \\
& +\lambda_{4}\left(\Phi_{1}^{\dagger} \Phi_{2}\right)\left(\Phi_{2}^{\dagger} \Phi_{1}\right)+\left[\frac{\lambda_{5}}{2}\left(\Phi_{1}^{\dagger} \Phi_{2}\right)^{2}+\text { H.c. }\right] .
\end{aligned}
$$

Here we focus on the $C P$-conserving case in which all $\lambda_{i}$ and $m_{12}^{2}$ are real. The two complex Higgs doublets have hypercharge $Y=1$ :

$$
\begin{aligned}
& \Phi_{1}=\left(\begin{array}{c}
\phi_{1}^{+} \\
\frac{1}{\sqrt{2}}\left(v_{1}+\phi_{1}^{0}+i a_{1}\right)
\end{array}\right), \\
& \Phi_{2}=\left(\begin{array}{c}
\phi_{2}^{+} \\
\frac{1}{\sqrt{2}}\left(v_{2}+\phi_{2}^{0}+i a_{2}\right)
\end{array}\right),
\end{aligned}
$$

where $v_{1}$ and $v_{2}$ are the electroweak vacuum expectation values (VEVs) with $v^{2}=v_{1}^{2}+v_{2}^{2}=(246 \mathrm{GeV})^{2}$ and $\tan \beta=v_{2} / v_{1}$. After spontaneous electroweak symmetry breaking, the remaining five physical Higgs particles are two neutral $C P$-even states $h$ and $H$, one neutral pseudoscalar $A$, and two charged scalars $H^{ \pm}$.

In the type-II 2HDM, the up-type fermions obtain masses from the $\Phi_{2}$ field, and the down-type fermions from the $\Phi_{1}$ field $[15,16]$. The Yukawa interactions are given by

$-\mathcal{L}=Y_{u 2} \bar{Q}_{L} \tilde{\Phi}_{2} u_{R}+Y_{d 1} \bar{Q}_{L} \Phi_{1} d_{R}+Y_{\ell 1} \bar{L}_{L} \Phi_{1} e_{R}+$ H.c.

where $Q_{L}^{T}=\left(u_{L}, d_{L}\right), L_{L}^{T}=\left(\nu_{L}, l_{L}\right), \tilde{\Phi}_{1,2}=i \tau_{2} \Phi_{1,2}^{*}$, and $Y_{u 2}, Y_{d 1}$, and $Y_{\ell 1}$ are $3 \times 3$ matrices in family space.

The Yukawa couplings of the neutral Higgs bosons normalized to the SM are given by

$y_{V}^{h}=\sin (\beta-\alpha), \quad y_{f}^{h}=\left[\sin (\beta-\alpha)+\cos (\beta-\alpha) \kappa_{f}\right]$,

$y_{V}^{H}=\cos (\beta-\alpha), \quad y_{f}^{H}=\left[\cos (\beta-\alpha)-\sin (\beta-\alpha) \kappa_{f}\right]$,

$y_{V}^{A}=0, \quad y_{A}^{f}=-i \kappa_{f}($ for $u), \quad y_{f}^{A}=i \kappa_{f}($ for $d, \ell)$,

with $\quad \kappa_{d}=\kappa_{\ell} \equiv-\tan \beta, \quad \kappa_{u} \equiv 1 / \tan \beta$,

where $\alpha$ is the mixing angle of the two $C P$-even Higgs bosons, and $V$ denotes $Z$ or $W$.

\section{B. Scalar dark matter}

Now we add a real singlet scalar $S$ to the type-II 2HDM, and the potential containing the $S$ field is written as

$$
\mathcal{V}_{S}=\frac{1}{2} S^{2}\left(\kappa_{1} \Phi_{1}^{\dagger} \Phi_{1}+\kappa_{2} \Phi_{2}^{\dagger} \Phi_{2}\right)+\frac{m_{0}^{2}}{2} S^{2}+\frac{\lambda_{S}}{4 !} S^{4} .
$$

The linear and cubic terms of the $S$ field are forbidden by a $Z_{2}^{\prime}$ symmetry, under which $S \rightarrow-S$. The $S$ is a possible DM candidate since it does not acquire a VEV. We can obtain the DM mass and the cubic interactions with the neutral Higgses from Eq. (5),

$$
\begin{aligned}
m_{S}^{2} & =m_{0}^{2}+\frac{1}{2} \kappa_{1} v^{2} \cos ^{2} \beta+\frac{1}{2} \kappa_{2} v^{2} \sin ^{2} \beta, \\
-\lambda_{h} v S^{2} h / 2 & \equiv-\left(-\kappa_{1} \sin \alpha \cos \beta+\kappa_{2} \cos \alpha \sin \beta\right) v S^{2} h / 2, \\
-\lambda_{H} v S^{2} H / 2 & \equiv-\left(\kappa_{1} \cos \alpha \cos \beta+\kappa_{2} \sin \alpha \sin \beta\right) v S^{2} H / 2 .
\end{aligned}
$$

\section{NUMERICAL CALCULATIONS}

\section{A. Parameter space}

In this paper, we discuss two different scenarios as follows.

Case A: The light $C P$-even Higgs boson $h$ is taken as the $125 \mathrm{GeV}$ Higgs, i.e., $m_{h}=125 \mathrm{GeV}$, and $H$ and $A$ are heavier than $130 \mathrm{GeV}$. Both $h$ and $H$ are the portals between the DM and SM sectors, and contribute to the DM interactions with the SM particles.

Case B: The heavy $C P$-even Higgs boson $H$ is taken as the $125 \mathrm{GeV}$ Higgs, i.e., $m_{H}=125 \mathrm{GeV}$. The light $C P$ even Higgs $h$ is the only portal between the DM and SM sectors. The DM mass is taken to be slightly below the Higgs resonance, $m_{h} / 2=(1.0-1.2) \times m_{S}$. In the calculation of the thermally averaged cross section, the kinetic energy of the DM is non-negligible in the early Universe. As a result, the resonant condition in DM pair annihilation can be met for $m_{S}$ slightly smaller than $m_{h} / 2$. The temperature at the present time is much lower than the freeze-out temperature, and the velocity of DM is much smaller than that in the early Universe. The resonant condition for present-day DM pair annihilation is hardly satisfied for $m_{S}$ slightly smaller than $m_{h} / 2$. Therefore, a very small coupling of the DM and the light Higgs is possible to obtain the correct relic density, and accommodate the limits of the direct and indirect detections of DM. The situation will become complicated if the $125 \mathrm{GeV}$ Higgs mediates the interactions between the DM and SM sectors simultaneously. Certainly, the coupling of the DM and the $125 \mathrm{GeV}$ Higgs is required to be small enough to satisfy the bound from the invisible decay of the $125 \mathrm{GeV}$ Higgs. For simplicity, we assume that the $125 \mathrm{GeV}$ Higgs has no coupling to DM in Case B, namely $\lambda_{H}=0$.

\section{B. Theoretical and experimental constraints}

In our calculations, to implement the constraints from the Higgs searches at the LHC, we employ SusHi [17] to compute the Higgs cross sections in gluon fusion and 
$b \bar{b}$-associated production at next-to-next-to-leading order (NNLO) in QCD. The results from SusHi might not be reasonable for a small Higgs mass. In this paper, $m_{h}$ is taken to be larger than $20 \mathrm{GeV}$, and the corresponding DM mass of Case B is larger than $10 \mathrm{GeV}$. The measurement of the branching fraction of $b \rightarrow s \gamma$ imposes the strongest limit on the charged Higgs mass of the type-II 2HDM, $m_{H^{ \pm}}>580 \mathrm{GeV}$ [18]. The $S, T$, and $U$ oblique parameters give stringent constraints on the mass spectrum of Higgses of the type-II 2HDM [19-21]. One of $m_{A}$ and $m_{H}$ is around $600 \mathrm{GeV}$, and another is allowed to have a wide mass range including low masses [19]. Therefore, to allow $h$ to be light enough, we fix $m_{A}=600 \mathrm{GeV}$ in Case B.

In our calculations, we consider the following observables and constraints.

(1) Theoretical constraints: The scalar potential of the model contains the potential of the type-II 2HDM and the potential of the DM sector. Vacuum stability, perturbativity, and tree-level unitarity impose constraints on the relevant parameters, which are discussed in detail in Refs. [3,9]. Here we employ the formulas from Refs. [3,9] to implement the theoretical constraints. Compared to Refs. [3,9], there are additional factors of $\frac{1}{2}$ in the $\kappa_{1}$ term and the $\kappa_{2}$ term of this paper.

(2) The oblique parameters: The $S, T$, and $U$ parameters can impose strong constraints on the mass spectrum of Higgses of 2HDM. 2HDMC [22] is employed to implement the constraints from the oblique parameters $(S, T, U)$. The allowed values for these parameters are [23],

$$
\begin{gathered}
S=-0.03 \pm 0.1, \quad T=0.01 \pm 0.12, \\
U=0.05 \pm 0.1 .
\end{gathered}
$$

(3) The flavor observables and $R_{b}$ : SuPERIso-3.4 [24] is employed to calculate $\operatorname{Br}\left(B \rightarrow X_{s} \gamma\right) . \Delta m_{B_{s}}$ is calculated following the formulas in Ref. [25]. Besides, we perform the constraints of bottom quarks produced in $Z$ decays, $R_{b}$, which is calculated using the formulas in Refs. [26,27]. The experimental data fpr $\operatorname{Br}\left(B \rightarrow X_{s} \gamma\right)$ [28], $\Delta m_{s}$ [28], and $R_{b}$ [29] are given as

$$
\begin{aligned}
\operatorname{Br}\left(B \rightarrow X_{s} \gamma\right) & =(3.32 \pm 0.16) \times 10^{-4}, \\
\Delta m_{s} & =17.757 \pm 0.021 \mathrm{ps}^{-1}, \\
R_{b} & =0.21474 \pm 0.00003 .
\end{aligned}
$$

(4) The global fit to the signal data of the $125 \mathrm{GeV}$ Higgs: Because the $125 \mathrm{GeV}$ Higgs couplings with the SM particles in this model can be modified compared to the SM, the SM-like decay modes will be corrected. In Case A, $h$ is the $125 \mathrm{GeV}$ Higgs, and the invisible decay $h \rightarrow S S$ is kinematically allowed,
TABLE I. Combined best-fit signal strengths $\hat{\mu}_{g g H+t t H}(Y)$ and $\hat{\mu}_{V B F+V H}(Y)$, and $a_{Y}, b_{Y}$, and $c_{Y}$ for the corresponding Higgs decay modes.

\begin{tabular}{lccccr}
\hline \hline$Y$ & $\hat{\mu}_{g g H+t t H}(Y)$ & $\hat{\mu}_{V B F+V H}(Y)$ & $a_{Y}$ & $b_{Y}$ & \multicolumn{1}{c}{$c_{Y}$} \\
\hline$\gamma \gamma$ & 1.16 & 1.05 & 18.29 & 4.43 & 6.60 \\
$Z Z^{*}$ & 1.42 & 0.47 & 10.18 & 1.37 & 0.92 \\
$W W^{*}$ & 0.98 & 1.38 & 24.54 & 3.69 & 7.11 \\
$\tau \tau$ & 1.06 & 1.12 & 3.36 & 1.84 & 8.72 \\
$b \bar{b}$ & 1.15 & 0.65 & 1.07 & 0.0 & 11.10 \\
\hline \hline
\end{tabular}

which will be strongly constrained by the experimental data of the $125 \mathrm{GeV}$ Higgs. In Case $\mathrm{B}, H$ is the $125 \mathrm{GeV}$ Higgs, and the invisible decay $H \rightarrow S S$ is absent since the HSS coupling is taken to be zero. However, the decay $H \rightarrow h h$ is kinematically allowed for $m_{h}<62.5 \mathrm{GeV}$. We perform the $\chi^{2}$ calculation for the signal strengths of the $125 \mathrm{GeV}$ Higgs in the $\mu_{g g F+t t h}(Y)$ and $\mu_{V B F+V h}(Y)$ channels with $Y$ denoting the decay modes $\gamma \gamma, Z Z, W W, \tau^{+} \tau^{-}$, and $b \bar{b}$,

$$
\begin{aligned}
\chi^{2}(Y)= & \left(\begin{array}{c}
\mu_{g g H+t t H}(Y)-\hat{\mu}_{g g H+t t H}(Y) \\
\mu_{V B F+V H}(Y)-\hat{\mu}_{V B F+V H}(Y)
\end{array}\right)^{T}\left(\begin{array}{ll}
a_{Y} & b_{Y} \\
b_{Y} & c_{Y}
\end{array}\right) \\
& \times\left(\begin{array}{c}
\mu_{g g H+t t H}(Y)-\hat{\mu}_{g g H+t t H}(Y) \\
\mu_{V B F+V H}(Y)-\hat{\mu}_{V B F+V H}(Y)
\end{array}\right) .
\end{aligned}
$$

$\hat{\mu}_{g g H+t t H}(Y)$ and $\hat{\mu}_{V B F+V H}(Y)$ are the best-fit values, and $a_{Y}, b_{Y}$, and $c_{Y}$ are the parameters of the ellipse, which are given by the combined ATLAS and CMS experiments [30]. These values are listed in Table I.

We pay particular attention to the surviving samples with $\chi^{2}-\chi_{\min }^{2} \leq 6.18$, where $\chi_{\min }^{2}$ denotes the minimum of $\chi^{2}$. These samples correspond to the $2 \sigma$ range in any two-dimensional plane of the model parameters when explaining the Higgs data.

In addition, ATLAS and CMS reported upper limits on the branching ratio of the invisible decay of the $125 \mathrm{GeV}$ Higgs. In our calculation we impose the constraints, $\operatorname{Br}(h \rightarrow S S)<0.34$ [30].

(5) The nonobservation of additional Higgs bosons: We employ HiggsBounds [31,32] to implement the exclusion constraints from the searches for the neutral and charged Higgses at LEP at the 95\% confidence level. Especially for Case B, the searches for a light Higgs at LEP can impose stringent constraints on the parameter space.

At the LHC, ATLAS and CMS have searched for an additional scalar via its decay into various SM channels and some exotic decays. For $g g \rightarrow A$ production in the type-II $2 \mathrm{HDM}$, the contributions of the $b$-quark loop interfere destructively with those of the top-quark loop. The cross section decreases with an increase of $\tan \beta$, reaches a minimum value for a moderate value of $\tan \beta$, and is dominated by 
TABLE II. The upper limits at $95 \%$ C.L. on the production cross section times branching ratio of $\tau^{+} \tau^{-}, \mu^{+} \mu^{-}, \gamma \gamma, W W$, and $Z Z$ considered in the $\varphi^{\prime}$ and $A$ searches at the LHC. Here $\varphi^{\prime}$ denotes the non-SM-like $C P$-even Higgs in the 2 HDM.

\begin{tabular}{|c|c|c|c|}
\hline Channel & Experiment & Mass range $[\mathrm{GeV}]$ & Luminosity \\
\hline$g g / b \bar{b} \rightarrow \varphi^{\prime} / A \rightarrow \tau^{+} \tau^{-}$ & ATLAS $8 \mathrm{TeV}$ [44] & $90-1000$ & $19.5-20.3 \mathrm{fb}^{-1}$ \\
\hline$g g / b \bar{b} \rightarrow \varphi^{\prime} / A \rightarrow \tau^{+} \tau^{-}$ & CMS $8 \mathrm{TeV}$ [45] & $90-1000$ & $19.7 \mathrm{fb}^{-1}$ \\
\hline$g g / b \bar{b} \rightarrow \varphi^{\prime} / A \rightarrow \tau^{+} \tau^{-}$ & ATLAS $13 \mathrm{TeV}$ [46] & $200-1200$ & $13.3 \mathrm{fb}^{-1}$ \\
\hline$g g / b \bar{b} \rightarrow \varphi^{\prime} / A \rightarrow \tau^{+} \tau^{-}$ & CMS $13 \mathrm{TeV}$ [47] & $90-3200$ & $12.9 \mathrm{fb}^{-1}$ \\
\hline$g g \rightarrow \varphi^{\prime} / A \rightarrow \tau^{+} \tau^{-}$ & CMS $13 \mathrm{TeV}$ [48] & $200-2250$ & $36.1 \mathrm{fb}^{-1}$ \\
\hline$b \bar{b} \rightarrow \varphi^{\prime} / A \rightarrow \tau^{+} \tau^{-}$ & CMS $13 \mathrm{TeV}$ [48] & $200-2250$ & $36.1 \mathrm{fb}^{-1}$ \\
\hline$b \bar{b} \rightarrow \varphi^{\prime} / A \rightarrow \tau^{+} \tau^{-}$ & CMS $8 \mathrm{TeV}$ [49] & $20-80$ & $19.7 \mathrm{fb}^{-1}$ \\
\hline$b \bar{b} \rightarrow \varphi^{\prime} / A \rightarrow \mu^{+} \mu^{-}$ & CMS $8 \mathrm{TeV}$ [50] & $25-60$ & $19.7 \mathrm{fb}^{-1}$ \\
\hline$p p \rightarrow \varphi^{\prime} / A \rightarrow \gamma \gamma$ & ATLAS $13 \mathrm{TeV}$ [51] & $200-2400$ & $15.4 \mathrm{fb}^{-1}$ \\
\hline$g g \rightarrow \varphi^{\prime} / A \rightarrow \gamma \gamma$ & $\mathrm{CMS} 8+13 \mathrm{TeV}$ [52] & $500-4000$ & $12.9 \mathrm{fb}^{-1}$ \\
\hline$g g \rightarrow \varphi^{\prime} / A \rightarrow \gamma \gamma+t \bar{t} \varphi^{\prime} / A\left(\varphi^{\prime} / A \rightarrow \gamma \gamma\right)$ & CMS $8 \mathrm{TeV}$ [53] & $80-110$ & $19.7 \mathrm{fb}^{-1}$ \\
\hline$g g \rightarrow \varphi^{\prime} / A \rightarrow \gamma \gamma+t \bar{t} \varphi^{\prime} / A\left(\varphi^{\prime} / A \rightarrow \gamma \gamma\right)$ & CMS $13 \mathrm{TeV}$ [53] & $70-110$ & $35.9 \mathrm{fb}^{-1}$ \\
\hline$V V \rightarrow \varphi^{\prime} \rightarrow \gamma \gamma+V \varphi^{\prime}\left(\varphi^{\prime} \rightarrow \gamma \gamma\right)$ & CMS $8 \mathrm{TeV}$ [53] & $80-110$ & $19.7 \mathrm{fb}^{-1}$ \\
\hline$V V \rightarrow \varphi^{\prime} \rightarrow \gamma \gamma+V \varphi^{\prime}\left(\varphi^{\prime} \rightarrow \gamma \gamma\right)$ & CMS $13 \mathrm{TeV}$ [53] & $70-110$ & $35.9 \mathrm{fb}^{-1}$ \\
\hline$g g / V V \rightarrow \varphi^{\prime} \rightarrow W^{+} W^{-}$ & ATLAS $8 \mathrm{TeV}$ [54] & $300-1500$ & $20.3 \mathrm{fb}^{-1}$ \\
\hline$g g / V V \rightarrow \varphi^{\prime} \rightarrow W^{+} W^{-}(\ell \nu \ell \nu)$ & ATLAS $13 \mathrm{TeV}$ [55] & $300-3000$ & $13.2 \mathrm{fb}^{-1}$ \\
\hline$g g \rightarrow \varphi^{\prime} \rightarrow W^{+} W^{-}(\ell \nu q q)$ & ATLAS $13 \mathrm{TeV}$ [56] & $500-3000$ & $13.2 \mathrm{fb}^{-1}$ \\
\hline$g g / V V \rightarrow \varphi^{\prime} \rightarrow W^{+} W^{-}(\ell \nu q q)$ & ATLAS $13 \mathrm{TeV}$ [57] & $200-3000$ & $36.1 \mathrm{fb}^{-1}$ \\
\hline$g g / V V \rightarrow \varphi^{\prime} \rightarrow W^{+} W^{-}(e \nu \mu \nu)$ & ATLAS $13 \mathrm{TeV}$ [58] & $200-3000$ & $36.1 \mathrm{fb}^{-1}$ \\
\hline$g g / V V \rightarrow \varphi^{\prime} \rightarrow Z Z$ & ATLAS $8 \mathrm{TeV}$ [59] & $160-1000$ & $20.3 \mathrm{fb}^{-1}$ \\
\hline$g g \rightarrow \varphi^{\prime} \rightarrow Z Z(\ell \ell \nu \nu)$ & ATLAS $13 \mathrm{TeV}$ [60] & $300-1000$ & $13.3 \mathrm{fb}^{-1}$ \\
\hline$g g \rightarrow \varphi^{\prime} \rightarrow Z Z(\nu \nu q q)$ & ATLAS $13 \mathrm{TeV}$ [61] & $300-3000$ & $13.2 \mathrm{fb}^{-1}$ \\
\hline$g g / V V \rightarrow \varphi^{\prime} \rightarrow Z Z(\ell \ell q q)$ & ATLAS $13 \mathrm{TeV}$ [61] & $300-3000$ & $13.2 \mathrm{fb}^{-1}$ \\
\hline$g g / V V \rightarrow \varphi^{\prime} \rightarrow Z Z(\ell \ell \ell \ell)$ & ATLAS $13 \mathrm{TeV}$ [62] & 200-3000 & $14.8 \mathrm{fb}^{-1}$ \\
\hline$g g / V V \rightarrow \varphi^{\prime} \rightarrow Z Z(\ell \ell \ell \ell+\ell \ell \nu \nu)$ & ATLAS $13 \mathrm{TeV}$ [63] & 200-2000 & $36.1 \mathrm{fb}^{-1}$ \\
\hline$g g / V V \rightarrow \varphi^{\prime} \rightarrow Z Z(\nu \nu q q+\ell \ell q q)$ & ATLAS $13 \mathrm{TeV}$ [64] & $300-5000$ & $36.1 \mathrm{fb}^{-1}$ \\
\hline
\end{tabular}

the $b$-quark loop for a large enough value of $\tan \beta$. The cross section of the $C P$-even Higgs in gluon fusion depends on $\sin (\beta-\alpha)$ in addition to the Higgs mass and $\tan \beta$. We use SusHi [17] to compute the Higgs cross sections in gluon fusion and $b \bar{b}$ associated production at NNLO in QCD. A complete list of the searches for additional Higgses considered by us is summarized in Table II and Table III where some channels are taken from Ref. [33]. References [34,35] showed that the LHC searches for the charged Higgs fail to constrain the model for $m_{H^{ \pm}}>500 \mathrm{GeV}$. Therefore, the search channels for the charged Higgs are not included in this paper.

(6) The DM observables: We use MICROMEGAs [36] to calculate the relic density and present-day DM pair annihilation. The model file is generated using FEYnRules [37]. For $10 \mathrm{GeV}<m_{S}<50 \mathrm{GeV}$, the DM will annihilate into $b \bar{b}$ dominantly in this model.
In this model, the elastic scattering of $S$ on a nucleon receives contributions from the process with $t$-channel exchange of $h$ and $H$ in Case A, and only $h$ exchange in Case B. If both $h$ and $H$ contribute to the DM interactions with nucleons, the spinindependent cross section is given by [38]

$$
\sigma_{p(n)}=\frac{\mu_{p(n)}^{2}}{4 \pi m_{S}^{2}}\left[f^{p(n)}\right]^{2},
$$

where $\mu_{p(n)}=\frac{m_{S} m_{p(n)}}{m_{S}+m_{p(n)}}$,

$f^{p(n)}=\sum_{q=u, d, s} f_{q}^{p(n)} \mathcal{C}_{S q} \frac{m_{p(n)}}{m_{q}}+\frac{2}{27} f_{g}^{p(n)} \sum_{q=c, b, t} \mathcal{C}_{S q} \frac{m_{p(n)}}{m_{q}}$,

with $\mathcal{C}_{S q}=\frac{\lambda_{h}}{m_{h}^{2}} m_{q} y_{q}^{h}+\frac{\lambda_{H}}{m_{H}^{2}} m_{q} y_{q}^{H}$. The values of the form factors $f_{q}^{p, n}$ and $f_{g}^{p, n}$ are extracted using MICROMEGAs [36]. 
TABLE III. The upper limits at 95\% C.L. on the production cross section times branching ratio for the channels of Higgs-pair and Higgs production in association with $Z$ at the LHC. Here $\varphi^{\prime}$ and $\varphi_{s}$ denote the non-SM-like $C P$-even Higgs and the $125 \mathrm{GeV}$ Higgs in the $2 \mathrm{HDM}$, respectively.

\begin{tabular}{|c|c|c|c|}
\hline Channel & Experiment & Mass range $[\mathrm{GeV}]$ & Luminosity \\
\hline$g g \rightarrow \varphi^{\prime} \rightarrow \varphi_{s} \varphi_{s} \rightarrow(\gamma \gamma)(b \bar{b})$ & CMS $8 \mathrm{TeV}[65]$ & $250-1100$ & $19.7 \mathrm{fb}^{-1}$ \\
\hline$g g \rightarrow \varphi^{\prime} \rightarrow \varphi_{s} \varphi_{s} \rightarrow(b \bar{b})(b \bar{b})$ & CMS $8 \mathrm{TeV}[66]$ & $270-1100$ & $17.9 \mathrm{fb}^{-1}$ \\
\hline$g g \rightarrow \varphi^{\prime} \rightarrow \varphi_{s} \varphi_{s} \rightarrow(b \bar{b})\left(\tau^{+} \tau^{-}\right)$ & CMS 8 TeV [67] & $260-350$ & $19.7 \mathrm{fb}^{-1}$ \\
\hline$g g \rightarrow \varphi^{\prime} \rightarrow \varphi_{s} \varphi_{s} \rightarrow(\gamma \gamma)(b \bar{b})$ & ATLAS $13 \mathrm{TeV}$ [68] & $275-400$ & $3.2 \mathrm{fb}^{-1}$ \\
\hline$g g \rightarrow \varphi^{\prime} \rightarrow \varphi_{s} \varphi_{s} \rightarrow(\gamma \gamma)(b \bar{b})$ & CMS 13 TeV [69] & $250-900$ & $2.7 \mathrm{fb}^{-1}$ \\
\hline$g g \rightarrow \varphi^{\prime} \rightarrow \varphi_{s} \varphi_{s} \rightarrow b \bar{b} b \bar{b}$ & ATLAS $13 \mathrm{TeV}$ [70] & $300-3000$ & $13.3 \mathrm{fb}^{-1}$ \\
\hline$g g \rightarrow \varphi^{\prime} \rightarrow \varphi_{s} \varphi_{s} \rightarrow(b \bar{b})\left(\tau^{+} \tau^{-}\right)$ & CMS $13 \mathrm{TeV}$ [71] & $250-900$ & $12.9 \mathrm{fb}^{-1}$ \\
\hline$g g \rightarrow \varphi^{\prime} \rightarrow \varphi_{s} \varphi_{s} \rightarrow b \bar{b} b \bar{b}$ & CMS $13 \mathrm{TeV}$ [72] & $750-3000$ & $35.9 \mathrm{fb}^{-1}$ \\
\hline$g g \rightarrow \varphi^{\prime} \rightarrow \varphi_{s} \varphi_{s} \rightarrow(b \bar{b})\left(\tau^{+} \tau^{-}\right)$ & CMS $13 \mathrm{TeV}$ [73] & $250-900$ & $35.9 \mathrm{fb}^{-1}$ \\
\hline$g g \rightarrow \varphi^{\prime} \rightarrow \varphi_{s} \varphi_{s} \rightarrow\left(W W^{*}\right)(\gamma \gamma)$ & ATLAS $13 \mathrm{TeV}$ [73] & $260-500$ & $13.3 \mathrm{fb}^{-1}$ \\
\hline$g g \rightarrow A \rightarrow \varphi_{s} Z \rightarrow\left(\tau^{+} \tau^{-}\right)(\ell \ell)$ & CMS $8 \mathrm{TeV}$ [67] & $220-350$ & $19.7 \mathrm{fb}^{-1}$ \\
\hline$g g \rightarrow A \rightarrow \varphi_{s} Z \rightarrow(b \bar{b})(\ell \ell)$ & CMS $8 \mathrm{TeV}$ [74] & $225-600$ & $19.7 \mathrm{fb}^{-1}$ \\
\hline$g g \rightarrow A \rightarrow \varphi_{s} Z \rightarrow\left(\tau^{+} \tau^{-}\right) Z$ & ATLAS $8 \mathrm{TeV}$ [75] & $220-1000$ & $20.3 \mathrm{fb}^{-1}$ \\
\hline$g g \rightarrow A \rightarrow \varphi_{s} Z \rightarrow(b \bar{b}) Z$ & ATLAS $8 \mathrm{TeV}$ [75] & $220-1000$ & $20.3 \mathrm{fb}^{-1}$ \\
\hline$g g / b \bar{b} \rightarrow A \rightarrow \varphi_{s} Z \rightarrow(b \bar{b}) Z$ & ATLAS $13 \mathrm{TeV}$ [76] & $200-2000$ & $3.2 \mathrm{fb}^{-1}$ \\
\hline$g g / b \bar{b} \rightarrow A \rightarrow \varphi_{s} Z \rightarrow(b \bar{b}) Z$ & ATLAS $13 \mathrm{TeV}$ [77] & $200-2000$ & $36.1 \mathrm{fb}^{-1}$ \\
\hline$g g \rightarrow \varphi_{s} \rightarrow A A / \varphi^{\prime} \varphi^{\prime} \rightarrow \tau^{+} \tau^{-} \tau^{+} \tau^{-}$ & ATLAS $8 \mathrm{TeV}$ [78] & $4-50$ & $20.3 \mathrm{fb}^{-1}$ \\
\hline$p p \rightarrow \varphi_{s} \rightarrow A A / \varphi^{\prime} \varphi^{\prime} \rightarrow \tau^{+} \tau^{-} \tau^{+} \tau^{-}$ & CMS 8 TeV [79] & $5-15$ & $19.7 \mathrm{fb}^{-1}$ \\
\hline$p p \rightarrow \varphi_{s} \rightarrow A A / \varphi^{\prime} \varphi^{\prime} \rightarrow\left(\mu^{+} \mu^{-}\right)(b \bar{b})$ & CMS $8 \mathrm{TeV}$ [79] & $25-62.5$ & $19.7 \mathrm{fb}^{-1}$ \\
\hline$p p \rightarrow \varphi_{s} \rightarrow A A / \varphi^{\prime} \varphi^{\prime} \rightarrow\left(\mu^{+} \mu^{-}\right)\left(\tau^{+} \tau^{-}\right)$ & CMS $8 \mathrm{TeV}[79]$ & $15-62.5$ & $19.7 \mathrm{fb}^{-1}$ \\
\hline$g g \rightarrow A\left(\varphi^{\prime}\right) \rightarrow \varphi^{\prime}(A) Z \rightarrow(b \bar{b})(\ell \ell)$ & CMS $8 \mathrm{TeV}[80]$ & $40-1000$ & $19.8 \mathrm{fb}^{-1}$ \\
\hline$g g \rightarrow A\left(\varphi^{\prime}\right) \rightarrow \varphi^{\prime}(A) Z \rightarrow\left(\tau^{+} \tau^{-}\right)(\ell \ell)$ & CMS $8 \mathrm{TeV}[80]$ & $20-1000$ & $19.8 \mathrm{fb}^{-1}$ \\
\hline
\end{tabular}

The Planck Collaboration reported the relic density of cold DM in the Universe, $\Omega_{c} h^{2}=$ $0.1198 \pm 0.0015$ [39]. The PandaX-II experiment (2017) imposed stronger constraints on the spin-independent DM-nucleon cross section than XENON (2017) for $m_{S}>100 \mathrm{GeV}$ [40,41]. In May of this year, the XENON Collaboration released an updated bound which increases current constraints on the cross section by a factor of $\sim 7$ for small DM mass and $\sim 2$ for large DM mass [42]. For $m_{S}<50 \mathrm{GeV}$, the bounds of XENON1T (2018) are much stronger than those of PandaX-II (2017). The Fermi-LAT searches for DM annihilation from $\mathrm{dSphs}$ gave the upper limits on the averaged cross sections of DM annihilation to $e^{+} e^{-}, \mu^{+} \mu^{-}, \tau^{+} \tau^{-}$, $u \bar{u}, b \bar{b}$, and $W W[43]^{*}$.

\section{RESULTS AND DISCUSSIONS}

\section{A. Case A}

In Fig. 1, we show $\lambda_{H}$ and $\lambda_{h}$ in Case $\mathrm{A}$ allowed by theoretical constraints, the oblique parameters, the signal data of the $125 \mathrm{GeV}$ Higgs, flavor observables, $R_{b}$, and the exclusion limits from searches for Higgs at LEP. The left panel shows that vacuum stability, perturbativity, unitarity, and the oblique parameters impose upper and lower limits on $\lambda_{H},-5<\lambda_{H}<7$. Because the invisible decay $h \rightarrow S S$ is kinematically allowed, the signal data of the $125 \mathrm{GeV}$ Higgs impose strong upper limits on $\left|\lambda_{h}\right|,\left|\lambda_{h}\right|<0.017$ $(0.022)$ for $m_{S}=10(50) \mathrm{GeV}$. However, the renormalization group analysis shows that the size of $\lambda_{H}$ increases with energy, and so it eventually meets a Landau pole at a higher energy scale, if $\lambda_{H}$ is too large at low energy [81]. Since this paper deals with the early Universe at high energy, we limit $\left|\lambda_{H}\right|$ to be smaller than 1.0 in the following discussions.

In Fig. 2, we project the surviving samples on the planes of $\tan \beta$ versus $m_{H}$ and $\lambda_{H}$ versus $m_{H}$ after imposing the "pre-LHC" constraints (denoting theory, the oblique parameters, flavor observables, $R_{b}$, and the exclusion limits from searches for Higgs at LEP), the signal data of the $125 \mathrm{GeV}$ Higgs, the relic density, and XENON1T (2018). Because the signal data of the $125 \mathrm{GeV}$ Higgs impose strong upper limits on $\left|\lambda_{h}\right|$, the DM interactions mediated by $H$ play a key role in the relic density. From Fig. 2, we find that the model can give the correct relic density for the heavy $C P$-even Higgs mass up to $650 \mathrm{GeV}$. With an increase of $m_{H}, \lambda_{H}$ and $\tan \beta$ are favored to have large values, which can enhance the couplings of $H S S$ and $H b \bar{b}$. 

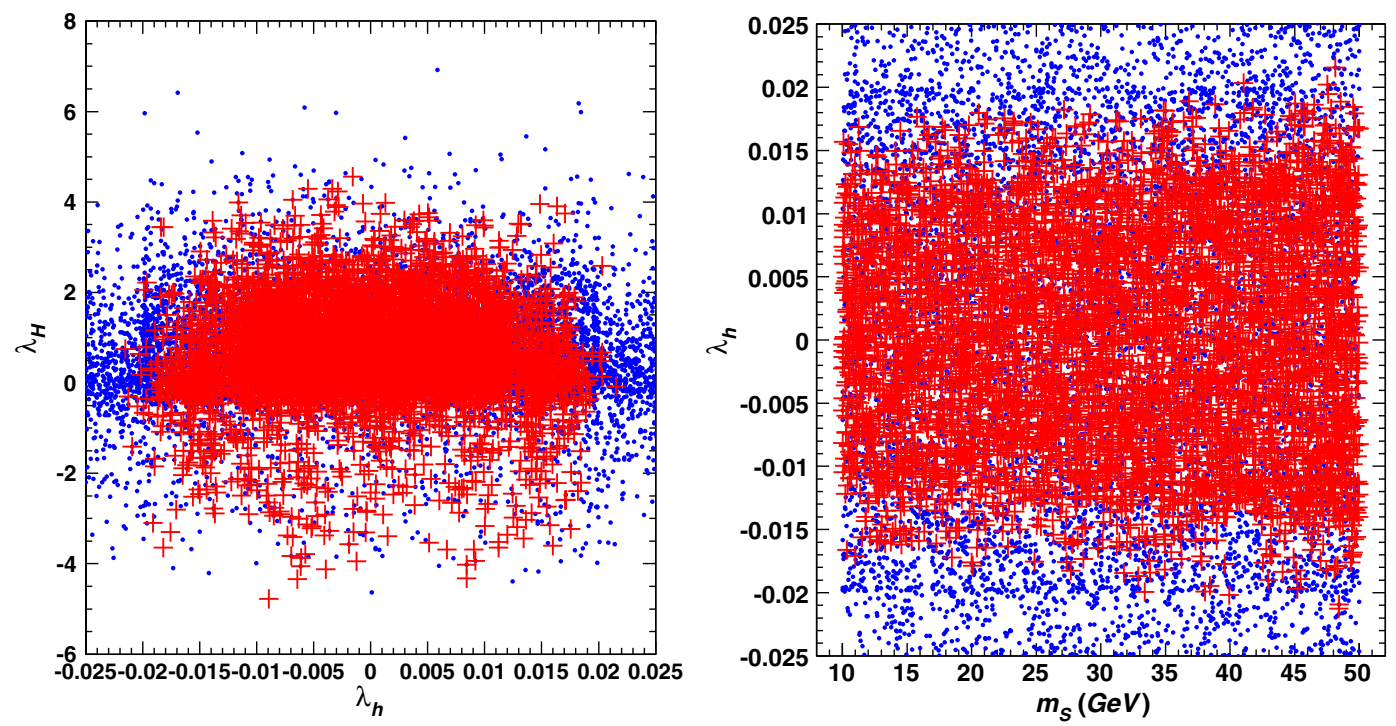

FIG. 1. In Case A, the surviving samples projected on the planes of $\lambda_{H}$ versus $\lambda_{h}$ and $\lambda_{h}$ versus $m_{S}$. All the samples are allowed by the constraints from the vacuum stability, perturbativity, unitarity, and the oblique parameters. The pluses (red) are also allowed by the joint constraints from the signal data of the $125 \mathrm{GeV}$ Higgs, the flavor observables, $R_{b}$, and the exclusion limits from searches for Higgs at LEP.

This is because the $H$-mediated annihilation amplitude is suppressed by its large mass, and the $H$ couplings to the $\mathrm{DM}$ and the SM particles are required to be large enough to obtain the correct annihilation rate. Most of the parameter spaces are excluded by XENON1T (2018), and only the narrow regions of $M_{H}<240 \mathrm{GeV}$ and $\tan \beta$ around 1.0 are allowed. For $\tan \beta$ around 1.0 and $|\sin (\beta-\alpha)|$ close to 1.0, $y_{d}^{H} / y_{u}^{H}$ approaches -1.0 . For such a case, the DM interactions with nucleons mediated by $H$ can have a large isospin violation, which may significantly weaken the bounds from XENON1T. Because $\tan \beta$ is restricted to be around 1.0, an appropriate value of $\lambda_{H}$ is required to obtain the correct relic density. For example, for the samples allowed by XENON1T (2018) in the right panel, $\left|\lambda_{H}\right|$ approaches 1.0 for $m_{H}$ around $240 \mathrm{GeV}$.

Figure 3 shows that the limits of the Fermi-LAT searches for DM annihilation from $\mathrm{dSphs}$ exclude the whole region of $10 \mathrm{GeV}<m_{S}<50 \mathrm{GeV}$, including the parameter space surviving from the XENON1T (2018) bounds. As $m_{S}$ decreases, the values of present-day DM pair annihilation
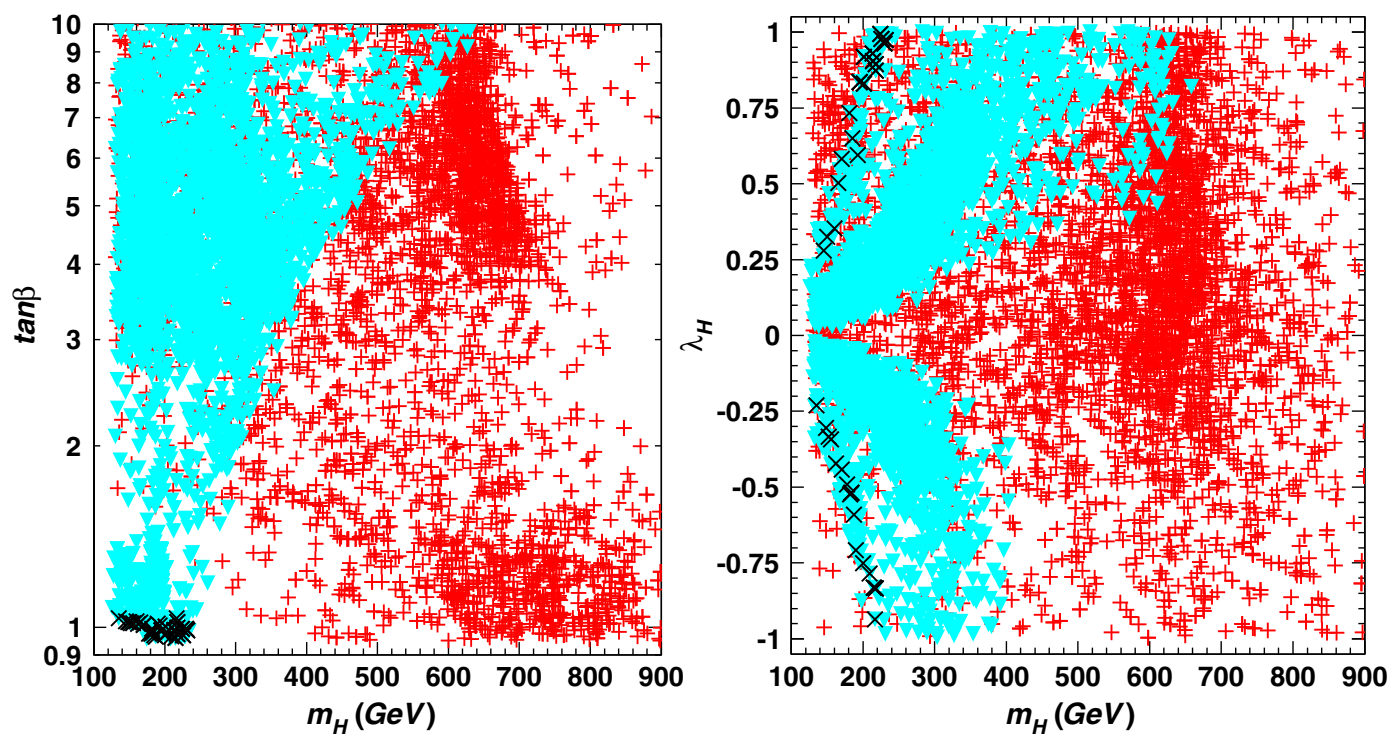

FIG. 2. In Case A, the surviving samples projected on the planes of $\tan \beta$ versus $m_{H}$ and $\lambda_{H}$ versus $m_{H}$. All the samples are allowed by the "pre-LHC" constraints and the signal data of the $125 \mathrm{GeV}$ Higgs. Also the inverted triangles (sky blue) are allowed by the relic density, and the crosses (black) are allowed by the relic density and the XENON1T (2018). 


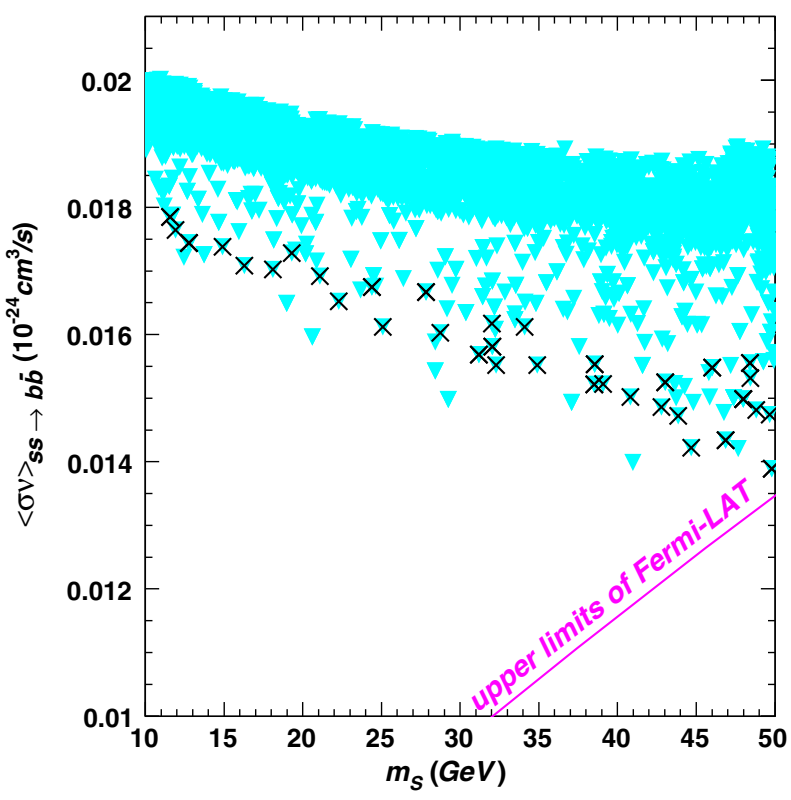

FIG. 3. The surviving samples projected on the planes of $\langle\sigma v\rangle_{S S \rightarrow b \bar{b}}$ versus $m_{S}$ in Case A. The meanings of the inverted triangles (sky blue) and crosses (black) are the same as in Fig. 2.

into $b \bar{b}$ in this model significantly exceed the Fermi-LAT upper limits.

\section{B. Case B}

Now we discuss Case $\mathrm{B}$ in which the heavy $C P$-even Higgs is the $125 \mathrm{GeV}$ Higgs. In Fig. 4, we project the surviving samples on the planes of $\tan \beta$ versus $m_{h}$ and $\sin (\beta-\alpha)$ versus $m_{h}$ after imposing the "pre-LHC" constraints, the signal data of the $125 \mathrm{GeV}$ Higgs, the searches for the additional Higgses at the LHC, the DM relic density, XENON1T (2018), and the Fermi-LAT searches for DM annihilation from dSphs. The left panel shows that the signal data of the $125 \mathrm{GeV}$ Higgs restrict $\tan \beta$ to be in the range of $1-1.5$ for $m_{h}<62 \mathrm{GeV}$. For such a range of $m_{h}$, the decay $H \rightarrow h h$ is kinematically open, and enhances the total width of the $125 \mathrm{GeV}$ Higgs. The width of $H \rightarrow h h$ is strongly constrained by the global fit to the signal data of the $125 \mathrm{GeV}$ Higgs, which easily satisfies the searches for a light Higgs via $H \rightarrow h h$ channels at the LHC. The right panel shows that $\sin (\beta-\alpha)$ imposes a lower bound for a given value of $m_{h}$. This is because the $h Z Z$ coupling is proportional to $\sin (\beta-\alpha)$, and a large absolute value of $\sin (\beta-\alpha)$ is excluded by the searches for a light Higgs via $e^{+} e^{-} \rightarrow Z h$ at LEP.

The left panel of Fig. 4 shows that the $g g \rightarrow A \rightarrow h Z$ channels at the LHC impose lower bounds on $\tan \beta$ for $53 \mathrm{GeV}<m_{h}<120 \mathrm{GeV}$ with $m_{A}$ being taken as $600 \mathrm{GeV}$, such as $\tan \beta>1.3$ for $m_{h}=55 \mathrm{GeV}, \tan \beta>2.3$ for $m_{h}=70 \mathrm{GeV}$, and $\tan \beta>2.7$ for $m_{h}=110 \mathrm{GeV}$. The $A h Z$ coupling is proportional to $\cos (\beta-\alpha)$ and not suppressed by $\sin (\beta-\alpha)$. However, the cross section of $g g \rightarrow A$ will sizably decrease with an increase of $\tan \beta$. The $b \bar{b} \rightarrow$ $h \rightarrow \tau^{+} \tau^{-}$channels impose upper limits on $\tan \beta$, and $\tan \beta>10$ is excluded for both $m_{h}<80 \mathrm{GeV}$ and $m_{h}>$ $90 \mathrm{GeV}$. In the range of $80 \mathrm{GeV}<m_{h}<90 \mathrm{GeV}$, there is no available experimental data for $b \bar{b} \rightarrow h \rightarrow \tau^{+} \tau^{-}$from ATLAS and CMS.

Figure 4 shows that a narrow region of $m_{h}$ around $60 \mathrm{GeV}$ and $m_{A}=600 \mathrm{GeV}$ is excluded by the joint constraints of the $125 \mathrm{GeV}$ Higgs signal data and the
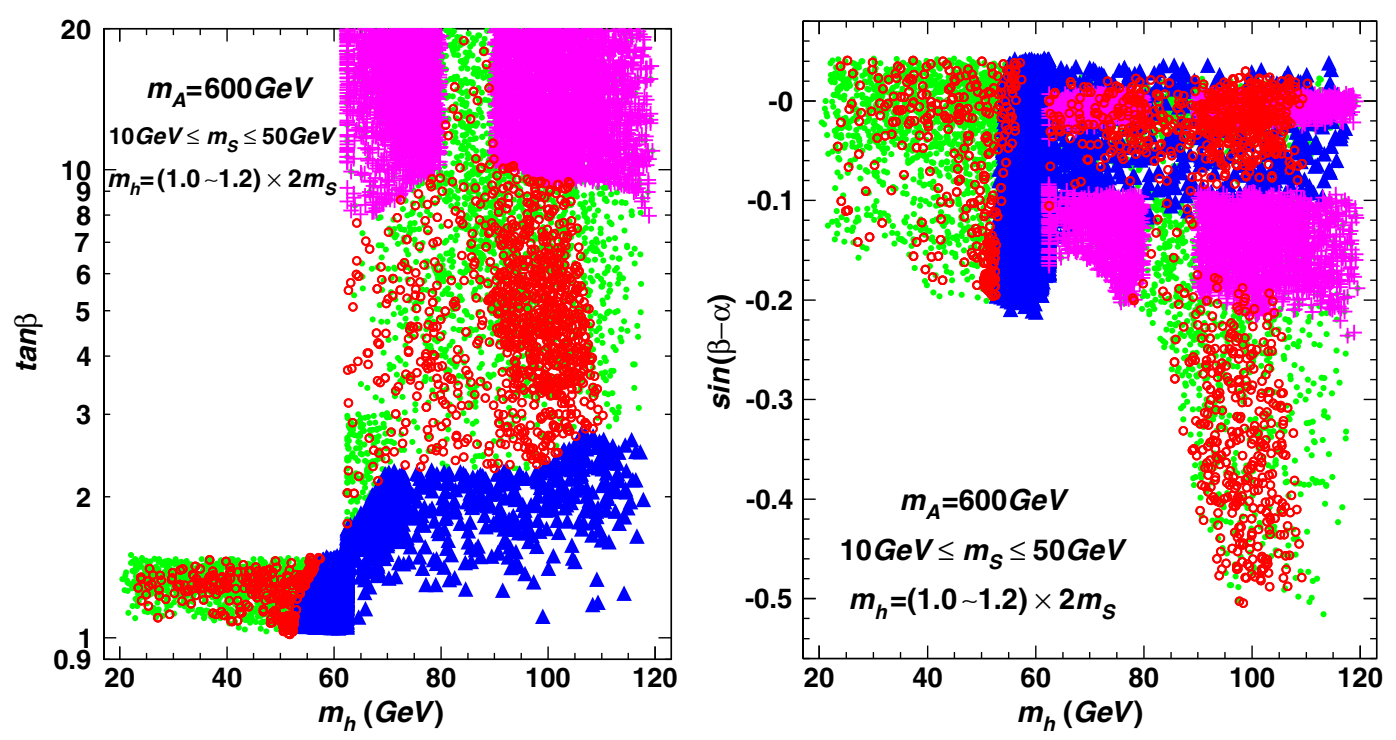

FIG. 4. The surviving samples projected on the planes of $\tan \beta$ versus $m_{h}$ and $\sin (\beta-\alpha)$ versus $m_{h}$ in Case B. All the samples are allowed by the constraints of "pre-LHC" and the signal data of the $125 \mathrm{GeV}$ Higgs. The pluses (pink) and the triangles (royal blue) are respectively excluded by the $h \rightarrow \tau^{+} \tau^{-}$and $A \rightarrow h Z$ channels at the LHC, and the other points are allowed by the two channels. The circles (red) are also allowed by the constraints from the relic density, XENON1T (2018), and Fermi-LAT. 

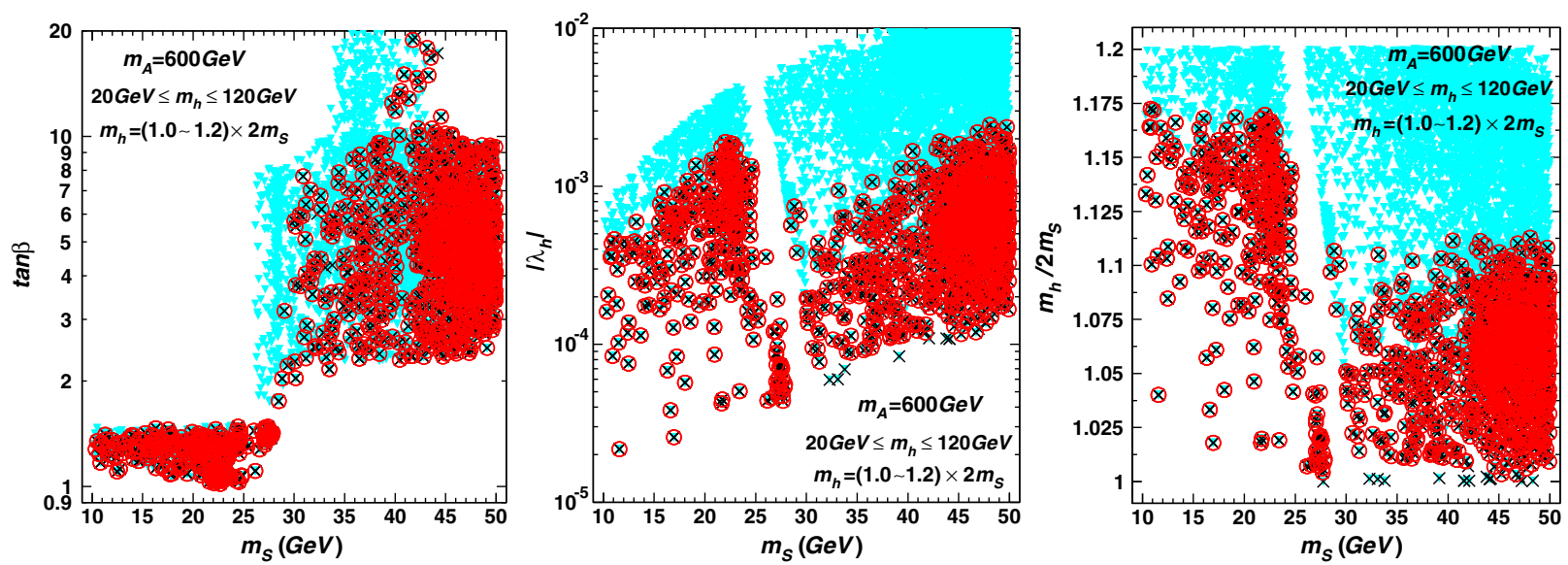

FIG. 5. The surviving samples projected on the planes of $\tan \beta$ versus $m_{S}, \lambda_{h}$ versus $m_{S}$, and $\frac{m_{h}}{2 m_{S}}$ versus $m_{S}$ in Case B. All the samples are allowed by the "pre-LHC" constraints, the signal data of the $125 \mathrm{GeV}$ Higgs, and the relic density. Also the crosses (black) are allowed by XENON1T (2018), and the circles (red) are allowed by XENON1T (2018) and Fermi-LAT.

$g g \rightarrow A \rightarrow h Z$ channels at the LHC. In the other region of $m_{h}$, DM with a mass of $10-50 \mathrm{GeV}$ is allowed by the constraints of the relic density, XENON1T (2018), and the Fermi-LAT searches for DM annihilation from dSphs. Certainly, the DM coupling with $h$ plays an important role.

In Fig. 5, we project the surviving samples on the planes of $\tan \beta$ versus $m_{S}, \lambda_{h}$ versus $m_{S}$, and $\frac{m_{h}}{2 m_{S}}$ versus $m_{S}$. From Fig. 5, we find that for appropriate values of $\tan \beta, \lambda_{h}$, and $m_{h}$, DM with a mass of $10-50 \mathrm{GeV}$ is allowed by the "preLHC" constraints, the signal data of the $125 \mathrm{GeV}$ Higgs, the searches for the additional Higgses at the LHC, the DM relic density, XENON1T (2018), and Fermi-LAT. The right panel shows that XENON1T (2018) excludes the region of $\frac{m_{h}}{2 m_{S}}>1.12$ and $30 \mathrm{GeV}<m_{S}<50 \mathrm{GeV}$. In such ranges, the kinetic energy of DM in the early Universe cannot offset the splitting of $m_{h}$ and $2 m_{S}$, and the resonant condition in the DM pair annihilation is not met. Therefore, a large $h S S$ coupling is required to obtain the correct relic density, which causes the spin-independent DM-nucleon cross section to exceed the upper limits of XENON1T (2018). Several points with $\frac{m_{h}}{2 m_{S}}$ very close to 1.0 are allowed by XENON1T (2018), but excluded by the Fermi-LAT limits. This is because the resonant condition for present-day DM pair annihilation is also satisfied for $\frac{m_{h}}{2 m_{S}}$ very close to 1.0 . The left panel shows that $\tan \beta$ is restricted to be in the range of $1.0-1.5$ for $10 \mathrm{GeV}<m_{s}<28 \mathrm{GeV}$. The middle panel shows that $\left|\lambda_{h}\right|$ is allowed to be as low as $10^{-5}$ because of the $h$ resonance contributions to DM pair annihilation.

In Fig. 6, we project the surviving samples on the planes of $\operatorname{Br}(h \rightarrow S S)$ versus $m_{h}$ and $\operatorname{Br}(h \rightarrow S S)$ versus $\frac{m_{h}}{2 m_{S}}$. Figure 6 shows that in the parameter space allowed by

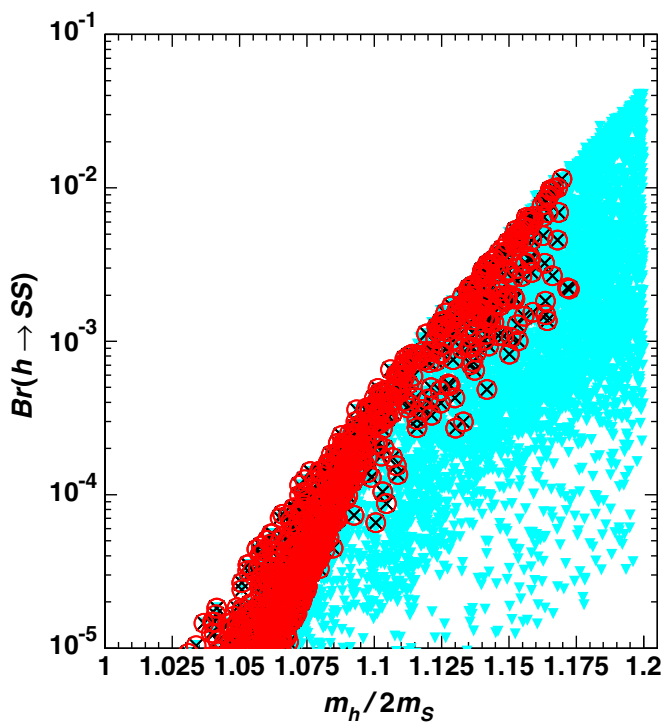

FIG. 6. Same as Fig. 5, but projected on the planes of $\operatorname{Br}(h \rightarrow S S)$ versus $m_{h}$ and $\operatorname{Br}(h \rightarrow S S)$ versus $\frac{m_{h}}{2 m_{S}}$. 
TABLE IV. The ranges of $m_{S}$ and several corresponding key parameters surviving all bounds with $\lambda_{H}=0$.

\begin{tabular}{lccc}
\hline \hline$m_{S}$ & $\tan \beta$ & $\left|\lambda_{h}\right|$ & $m_{h} / 2 m_{S}$ \\
\hline $10-28 \mathrm{GeV}$ & $1.0-1.5$ & $2 \times 10^{-5}-2 \times 10^{-3}$ & $1.004-1.175$ \\
$28-39 \mathrm{GeV}$ & $1.7-10$ & $7 \times 10^{-5}-1.5 \times 10^{-3}$ & $1.01-1.11$ \\
$39-44 \mathrm{GeV}$ & $2.3-20$ & $1 \times 10^{-4}-2 \times 10^{-3}$ & $1.01-1.113$ \\
$44-50 \mathrm{GeV}$ & $2.3-11$ & $1 \times 10^{-4}-2.6 \times 10^{-3}$ & $1.004-1.113$ \\
\hline \hline
\end{tabular}

XENON1T (2018) and Fermi-LAT, $\operatorname{Br}(h \rightarrow S S)$ is smaller than $2 \%$ for $m_{h}<60 \mathrm{GeV}$ and smaller than $0.1 \%$ for $60 \mathrm{GeV}<m_{h}<120 \mathrm{GeV}$. The current searches for DM at the LHC do not impose constraints on the parameter space.

As we discussed above, DM with a mass below $50 \mathrm{GeV}$ is excluded in all parameter spaces of Case A, and allowed in some parameter spaces of Case B. In Table IV, we simply summarize the ranges of $m_{S}$ and several corresponding key parameters surviving all bounds.

\section{CONCLUSION}

The type-II 2HDM with scalar DM provides a WIMPDM candidate economically. Some recent studies did not find the parameter space where DM with a mass below $50 \mathrm{GeV}$ is allowed by the constraints from the Higgs searches at the LHC and DM experiments. In this paper, we examined DM with a mass below $50 \mathrm{GeV}$ in the model after imposing the theoretical and experimental constraints.

We first discussed a general scenario in which both $C P$-even Higgses ( $h$ and $H$ ) are portals between the DM and SM sectors, and the $C P$-odd Higgs $(A)$ and $H$ are heavier than $130 \mathrm{GeV}$. We found that DM with a mass of $10-50 \mathrm{GeV}$ is disfavored by the joint constraints of the $125 \mathrm{GeV}$ Higgs signal data, the relic density, XENON1T (2018), and Fermi-LAT.

Next, we considered a special scenario in which the heavy $C P$-even Higgs is taken as the $125 \mathrm{GeV}$ Higgs, and the light $C P$-even Higgs is the only portal between the DM and SM sectors. The DM mass is slightly below the Higgs resonance, $m_{h} / 2=(1.0 \sim 1.2) \times m_{S}$. We found that the signal data of the $125 \mathrm{GeV}$ Higgs restrict $\tan \beta$ to be in the range of $1-1.5$ for $m_{h}<62 \mathrm{GeV}$. The $g g \rightarrow A \rightarrow h Z$ channels at the LHC give lower bounds on $\tan \beta$ for $53 \mathrm{GeV}<$ $m_{h}<120 \mathrm{GeV}$ and $m_{A}=600 \mathrm{GeV}$. The $b \bar{b} \rightarrow h \rightarrow \tau^{+} \tau^{-}$ channels impose upper limits on $\tan \beta$, and $\tan \beta>10$ is excluded for both $m_{h}<80 \mathrm{GeV}$ and $m_{h}>90 \mathrm{GeV}$. For appropriate values of $\tan \beta, \lambda_{h}$, and $m_{h}$, DM with a mass of $10-50 \mathrm{GeV}$ is allowed by the "pre-LHC" constraints, the signal data of the $125 \mathrm{GeV}$ Higgs, the searches for the additional Higgses at the LHC, the DM relic density, XENON1T (2018), and Fermi-LAT. For example, $\tan \beta$ is restricted to be in the range of $1.0-1.5$ for $10 \mathrm{GeV}<m_{s}<28 \mathrm{GeV}$, and $\frac{m_{h}}{2 m_{S}}>1.12$ is excluded for $30 \mathrm{GeV}<m_{S}<50 \mathrm{GeV}$.

\section{ACKNOWLEDGMENTS}

We thank Lei Feng for helpful discussions. This work is supported by the National Natural Science Foundation of China under grant No. 11575152, and the Natural Science Foundation of Shandong province (ZR2017MA004 and ZR2017JL002).
[1] V. Silveira and A. Zee, Phys. Lett. B 161, 136 (1985).

[2] L. Feng, S. Profumo, and L. Ubaldi, J. High Energy Phys. 03 (2015) 045.

[3] X.-G. He and J. Tandean, J. High Energy Phys. 12 (2016) 074.

[4] T. D. Lee, Phys. Rev. D 8, 1226 (1973).

[5] X.-G. He, T. Li, X.-Q. Li, J. Tandean, and H.-C. Tsai, Phys. Rev. D 79, 023521 (2009).

[6] X.-G. He and J. Tandean, Phys. Rev. D 88, 013020 (2013).

[7] Y. Cai and T. Li, Phys. Rev. D 88, 115004 (2013).

[8] L. Wang and X.-F. Han, Phys. Lett. B 739, 416 (2014).

[9] A. Drozd, B. Grzadkowski, J. F. Gunion, and Y. Jiang, J. High Energy Phys. 11 (2014) 105.

[10] A. Drozd, B. Grzadkowski, J. F. Gunion, and Y. Jiang, J. Cosmol. Astropart. Phys. 10 (2016) 040.

[11] T. Alanne, K. Kainulainen, K. Tuominen, and V. Vaskonen, J. Cosmol. Astropart. Phys. 08 (2016) 057.

[12] L. Wang, R. Shi, and X.-F. Han, Phys. Rev. D 96, 115025 (2017).
[13] N. Chen, Z. Kang, and J. Li, Phys. Rev. D 95, 015003 (2017).

[14] R. A. Battye, G. D. Brawn, and A. Pilaftsis, J. High Energy Phys. 08 (2011) 020.

[15] H. E. Haber, G. L. Kane, and T. Sterling, Nucl. Phys. B161, 493 (1979).

[16] J. F. Donoghue and L. F. Li, Phys. Rev. D 19, 945 (1979).

[17] R. V. Harlander, S. Liebler, and H. Mantler, Comput. Phys. Commun. 184, 1605 (2013).

[18] Heavy Flavor Averaging Group, Eur. Phys. J. C 77, 895 (2017); M. Misiak and M. Steinhauser, Eur. Phys. J. C 77, 201 (2017).

[19] L. Wang, F. Zhang, and X.-F. Han, Phys. Rev. D 95, 115014 (2017).

[20] M. Gorbahn, J. M. No, and V. Sanz, J. High Energy Phys. 10 (2015) 036.

[21] F. Kling, J. M. No, and S. Su, J. High Energy Phys. 09 (2016) 093. 
[22] D. Eriksson, J. Rathsman, and O. Stål, Comput. Phys. Commun. 181, 189 (2010).

[23] K. A. Olive et al. (Particle Data Group), Chin. Phys. C 38, 090001 (2014).

[24] F. Mahmoudi, Comput. Phys. Commun. 180, 1579 (2009).

[25] C. Q. Geng and J. N. Ng, Phys. Rev. D 38, 2857 (1988); 41, 1715(E) (1990).

[26] H. E. Haber and H. E. Logan, Phys. Rev. D 62, 015011 (2000).

[27] G. Degrassi and P. Slavich, Phys. Rev. D 81, 075001 (2010).

[28] Y. Amhis et al. (Heavy Flavor Averaging Group), Eur. Phys. J. C 77, 895 (2017).

[29] ALEPH, DELPHI, L3, OPAL, SLD, LEP Electroweak Working Group, SLD Electroweak Group, and SLD Heavy Flavor Group Collaborations, Phys. Rep. 427, 257 (2006).

[30] ATLAS and CMS Collaborations, J. High Energy Phys. 08 (2016) 045.

[31] P. Bechtle, O. Brein, S. Heinemeyer, G. Weiglein, and K. E. Williams, Comput. Phys. Commun. 181, 138 (2010).

[32] P. Bechtle, O. Brein, S. Heinemeyer, O. Stål, T. Stefaniak, G. Weiglein, and K. E. Williams, Eur. Phys. J. C 74, 2693 (2014).

[33] R. K. Barman, B. Bhattacherjee, A. Choudhury, D. Chowdhury, J. Lahiri, and S. Ray, arXiv:1608.02573.

[34] S. Moretti, Proc. Sci., CHARGED2016 (2016) 014, [arXiv:1612.02063].

[35] A. Arbey, F. Mahmoudi, O. Stal, and T. Stefaniak, Eur. Phys. J. C 78, 182 (2018).

[36] G. Belanger, F. Boudjema, A. Pukhov, and A. Semenov, Comput. Phys. Commun. 185, 960 (2014).

[37] A. Alloul, N. D. Christensen, C. Degrande, C. Duhr, and B. Fuks, Comput. Phys. Commun. 185, 2250 (2014).

[38] G. Jungman, M. Kamionkowski, and K. Griest, Phys. Rep. 267, 195 (1996); M. A. Shifman, A. I. Vainshtein, and V. I. Zakharov, Phys. Lett. B 78, 443 (1978).

[39] Planck Collaboration, Astron. Astrophys. 594, A24 (2016).

[40] PandaX Collaboration, Phys. Rev. Lett. 119, 181302 (2017).

[41] E. Aprile et al. (XENON Collaboration), Phys. Rev. Lett. 119, 181301 (2017).

[42] E. Aprile et al. (XENON Collaboration), arXiv:1805.12562.

[43] Fermi-LAT Collaboration, Phys. Rev. Lett. 115, 231301 (2015).

[44] G. Aad et al. (ATLAS Collaboration), J. High Energy Phys. 11 (2014) 056.

[45] CMS Collaboration, CERN Report No. CMS-PAS-HIG-14029.

[46] ATLAS Collaboration, CERN Report No. ATLAS-CONF2016-085.

[47] CMS Collaboration, CERN Report No. CMS-PAS-HIG-16037.

[48] ATLAS Collaboration, J. High Energy Phys. 01 (2018) 055.

[49] CMS Collaboration, Phys. Lett. B 758, 296 (2016).

[50] CMS Collaboration, CERN Report No. CMS-PAS-HIG-15009 .
[51] ATLAS Collaboration, CERN Report No. ATLAS-CONF2016-059.

[52] CMS Collaboration, CERN Report No. CMS-PAS-EXO16-027.

[53] CMS Collaboration, CERN Report No. CMS-PAS-HIG-17013.

[54] G. Aad et al. (ATLAS Collaboration), J. High Energy Phys. 01 (2016) 032.

[55] ATLAS Collaboration, CERN Report No. ATLAS-CONF2016-074.

[56] ATLAS Collaboration, CERN Report No. ATLAS-CONF2016-062.

[57] ATLAS Collaboration, J. High Energy Phys. 03 (2018) 042.

[58] ATLAS Collaboration, Eur. Phys. J. C 78, 24 (2018).

[59] G. Aad et al. (ATLAS Collaboration), Eur. Phys. J. C 76, 45 (2016).

[60] ATLAS Collaboration, Report No. ATLAS-CONF-2016056.

[61] ATLAS Collaboration, Report No. ATLAS-CONF-2016082 .

[62] ATLAS Collaboration, Report No. ATLAS-CONF-2016079.

[63] ATLAS Collaboration, Eur. Phys. J. C 78, 293 (2018).

[64] ATLAS Collaboration, J. High Energy Phys. 03 (2018) 009.

[65] V. Khachatryan et al. (CMS Collaboration), Phys. Rev. D 94, 052012 (2016).

[66] V. Khachatryan et al. (CMS Collaboration), Phys. Lett. B 749, 560 (2015).

[67] V. Khachatryan et al. (CMS Collaboration), Phys. Lett. B 755, 217 (2016).

[68] ATLAS Collaboration, Report No. ATLAS-CONF-2016004.

[69] V. Khachatryan et al. (CMS Collaboration), Report No. CMS-PAS-HIG-16-032.

[70] ATLAS Collaboration, Report No. ATLAS-CONF-2016049.

[71] CMS Collaboration, Report No. CMS-PAS-HIG-16-029.

[72] CMS Collaboration, Phys. Lett. B 781, 244 (2018).

[73] CMS Collaboration, Phys. Lett. B 778, 101 (2018).

[74] V. Khachatryan et al. (CMS Collaboration), Phys. Lett. B 748, 221 (2015).

[75] G. Aad et al. (ATLAS Collaboration), Phys. Lett. B 744, 163 (2015).

[76] ATLAS Collaboration, Report No. ATLAS-CONF-2016015.

[77] ATLAS Collaboration, J. High Energy Phys. 03 (2018) 174.

[78] ATLAS Collaboration, Phys. Rev. D 92, 052002 (2015).

[79] CMS Collaboration, J. High Energy Phys. 10 (2017) 076.

[80] V. Khachatryan et al. (CMS Collaboration), Phys. Lett. B 759, 369 (2016).

[81] I. Chakraborty and A. Kundu, Phys. Rev. D 87, 055015 (2013); B. Kyae and C. S. Shin, Phys. Rev. D 88, 015011 (2013). 